\title{
The effect of 8 or 5 years of denosumab treatment in postmenopausal women with osteoporosis: results from the FREEDOM Extension study
}

\author{
S. Papapoulos ${ }^{1}$ - K. Lippuner ${ }^{2}$ - C. Roux ${ }^{3}$ - C. J. F. Lin ${ }^{4}$ - D. L. Kendler ${ }^{5}$. \\ E. M. Lewiecki ${ }^{6}$ - M. L. Brandi ${ }^{7}$ - E. Czerwiński ${ }^{8}$ - E. Franek ${ }^{9}$ P. Lakatos ${ }^{10}$ • \\ C. Mautalen ${ }^{11} \cdot$ S. Minisola ${ }^{12}$ J. Y. Reginster ${ }^{13} \cdot$ S. Jensen ${ }^{14} \cdot$ N. S. Daizadeh ${ }^{4}$. \\ A. Wang ${ }^{4} \cdot$ M. Gavin ${ }^{4}$ - C. Libanati ${ }^{4}$ - R. B. Wagman ${ }^{4}$ - H. G. Bone ${ }^{15}$
}

Received: 15 December 2014 / Accepted: 2 July 2015 /Published online: 23 July 2015

(C) The Author(s) 2015. This article is published with open access at Springerlink.com

\begin{abstract}
Summary The FREEDOM study and its Extension provide long-term information about the effects of denosumab for the treatment of postmenopausal osteoporosis. Treatment for up to 8 years was associated with persistent reduction of bone turnover, continued increases in bone mineral density, low fracture incidence, and a favorable benefit/risk profile.

Introduction This study aims to report the results through year 5 of the FREEDOM Extension study, representing up to 8 years of continued denosumab treatment in postmenopausal women with osteoporosis.

Methods Women who completed the 3-year FREEDOM study were eligible to enter the 7-year open-label FREEDOM Extension in which all participants are scheduled to receive denosumab, since placebo assignment was discontinued for ethical reasons. A total of 4550 women enrolled in the Extension (2343 long-term; 2207 cross-over). In
\end{abstract}

this analysis, women in the long-term and cross-over groups received denosumab for up to 8 and 5 years, respectively. Results Throughout the Extension, sustained reduction of bone turnover markers (BTMs) was observed in both groups. In the long-term group, mean bone mineral density (BMD) continued to increase significantly at each time point measured, for cumulative 8-year gains of 18.4 and $8.3 \%$ at the lumbar spine and total hip, respectively. In the cross-over group, mean BMD increased significantly from the Extension baseline for 5-year cumulative gains of 13.1 and $6.2 \%$ at the lumbar spine and total hip, respectively. The yearly incidence of new vertebral and nonvertebral fractures remained low in both groups. The incidence of adverse and serious adverse events did not increase over time. Through Extension year 5, eight events of osteonecrosis of the jaw and two events of atypical femoral fracture were confirmed.

Electronic supplementary material The online version of this article (doi:10.1007/s00198-015-3234-7) contains supplementary material, which is available to authorized users.

S. Papapoulos

M.V.Iken@lumc.nl

1 Center for Bone Quality, Leiden University Medical Center, Albinusdreef 2, 2333 ZA Leiden, The Netherlands

2 Berne University Hospital and University, Berne, Switzerland

3 Paris Descartes University, Paris, France

4 Amgen Inc, Thousand Oaks, CA, USA

5 University of British Columbia, Vancouver, BC, Canada

6 New Mexico Clinical Research \& Osteoporosis Center, Albuquerque, NM, USA
University of Florence, Florence, Italy

8 Krakow Medical Center, Krakow, Poland

9 Mossakowski Medical Research Centre, Polish Academy of Sciences, Warsaw, Poland

10 Semmelweis University, Budapest, Hungary

11 Centro de Osteopatias Medicas, Buenos Aires, Argentina

12 Sapienza University, Rome, Italy

13 University of Liège, Liège, Belgium

14 Center for Clinical and Basic Research, Ballerup, Denmark

15 Michigan Bone \& Mineral Clinic, Detroit, MI, USA 
Conclusions Denosumab treatment for up to 8 years was associated with persistent reductions of BTMs, continued BMD gains, low fracture incidence, and a consistent safety profile.

Keywords Bone mineral density . Clinical trial . Denosumab $\cdot$ Fracture $\cdot$ Osteoporosis $\cdot$ Safety

\section{Introduction}

We have previously reported the results of up to 6 years of continuous denosumab therapy in women with postmenopausal osteoporosis in the 3-year FREEDOM study [1] and its ongoing 7-year Extension [2,3]. These data have supported a favorable benefit/risk profile of denosumab over time. We observed maintenance of the effect of denosumab on bone turnover that was associated with low fracture incidence, progressive and significant increases in bone mineral density (BMD) at all sites measured, and a well-maintained safety profile with exposure for up to 6 years.

Bone turnover and BMD are pharmacologically modifiable risk factors for fracture in patients with osteoporosis. Data from the FREEDOM study demonstrated that gains in BMD with denosumab treatment for up to 3 years were associated with increased estimated bone strength [4], which can account for much of the effect of denosumab in reducing fracture risk [5]. It is of considerable scientific and clinical interest to extend these observations.

The Extension study, which will ultimately result in up to 10 years of denosumab exposure, offers an opportunity to evaluate how safety and efficacy assessments trend over time in an aging population of postmenopausal women with osteoporosis. Here, we report the year-by-year safety and efficacy of continued denosumab therapy through the fifth year of the Extension to the denosumab phase 3 pivotal fracture study, FREEDOM, for a total of up to 8 years of treatment.

\section{Materials and methods}

\section{Study design}

The FREEDOM pivotal study (ClinicalTrials.gov: NCT00089791) and the Extension designs (ClinicalTrials.gov: NCT00523341) have been described elsewhere $[1,2]$ and are summarized in Online Resource Fig. S1. Briefly, postmenopausal women aged 60-90 years with a lumbar spine or total hip BMD T-score less than -2.5 at either site but -4.0 or greater at both sites were eligible to enroll in FREEDOM. Participants were randomized to receive placebo or $60 \mathrm{mg}$ denosumab (Prolia ${ }^{\circledR}$; Amgen Inc., Thousand Oaks, CA, USA) subcutaneously (s.c.) every 6 months for 3 years. All participants were instructed to take calcium $(\geq 1 \mathrm{~g})$ and vitamin $\mathrm{D}$ ( $\geq 400 \mathrm{IU})$ daily.
All women who completed the FREEDOM study (i.e., completed their 3-year visit) in either the denosumab or placebo arm, did not discontinue investigational product, and did not miss $>1$ dose of investigational product were eligible to enter the Extension where all participants are scheduled to receive openlabel $60 \mathrm{mg}$ denosumab s.c. every 6 months ( \pm 1 month) with daily calcium and vitamin D supplementation. The study is ongoing with preplanned data analyses performed at intervals as part of continuing pharmacovigilance. The data reported here include the first 5 years of the Extension and represent up to 8 years of denosumab exposure for women who received 3 years of denosumab in FREEDOM and continued in the Extension (long-term group), and up to 5 years of denosumab exposure for women who received 3 years of placebo in FREEDOM and transitioned to denosumab in the Extension (cross-over group).

The study protocol was approved by an institutional review board or ethics committee for each institutional site and all participants provided written informed consent. Representatives of the sponsor, Amgen Inc., designed the study with investigators, and statistical analyses were conducted according to a prespecified statistical analysis plan.

\section{Study procedures}

Study visits were scheduled at the Extension baseline (corresponding to the end of the FREEDOM parent study) and every 6 months for 7 years during the Extension.

Concentrations of serum C-terminal telopeptide of type 1 collagen (CTx; Nordic Bioscience Diagnostics A/S, Herlev, Denmark) and procollagen type $1 \mathrm{~N}$-terminal propeptide (P1NP; Orion Diagnostica Oy, Espoo, Finland) were measured by PPD (PPD Development, Richmond, VA, USA) and LabCorp (LabCorp Burlington, Burlington, NC, USA), respectively, using fasting (overnight), predose morning serum samples collected from a subset of women who participated in the FREEDOM bone turnover marker (BTM) substudy and consented to continue in the Extension. At year 2 of the Extension, the protocol was amended to increase the number of participants in the substudy. Evaluation of these additional participants began at year 3 of the Extension. After the publication of data from the first 2 years of the Extension [2], additional P1NP samples from the second year were identified in storage by the central laboratory. P1NP levels were measured in these samples, and the data are included in the current analysis. Consistent with previous reports, undetectable values were imputed using the corresponding assay's established lower limit of quantification (CTx, $0.049 \mathrm{ng} / \mathrm{mL}$; P1NP, $10 \mu \mathrm{g} / \mathrm{L}$ ) [6].

BMD measurements were performed by dual-energy X-ray absorptiometry (DXA) at the lumbar spine and hip in all women and at the 1/3 radius in a subset of women at the Extension baseline and Extension years 1, 2, 3, and 5. All DXA scans 
were centrally read by BioClinica (previously known as Synarc Inc.).

Vertebral fractures were also identified by a central facility (BioClinica) using the Genant semiquantitative grading scale [7] from thoracic and lumbar lateral radiographs obtained at the Extension baseline and Extension years 2, 3, and 5. Prevalent vertebral fractures at Extension baseline and new vertebral fractures were defined as described previously [2]. Clinical vertebral and nonvertebral fractures required confirmation by diagnostic imaging or a radiologist's report. Highforce trauma or pathological clinical vertebral and nonvertebral fractures were excluded, as described previously [1]. In this report, hip fractures were defined as those at the femoral neck or intertrochanteric region; previously, subtrochanteric fractures were also included [1-3].

Every 3 months, participants were queried about adverse events (AEs), the occurrence of a clinical fracture, and concomitant medications by the study site staff. Potential events of osteonecrosis of the jaw (ONJ) were reviewed by an independent, blinded, external adjudication committee, as described previously [3]. During year 3 of the Extension, an adjudication process was initiated to review potential events of atypical femoral fracture (AFF). Available X-ray images of femoral fractures that occurred were reviewed by a panel at the central radiographic vendor site (BioClinica); femoral fractures that occurred prior to year 3 were retrospectively reviewed. The major criteria established by the American Society for Bone and Mineral Research 2010 Task Force for AFF [8] were used for adjudication by this committee: fracture location (subtrochanteric, including the femur diaphysis); fracture type (non-comminuted, simple transverse, or short oblique); no or minimal trauma; and the absence of any evidence of malignancy, prosthesis, pins, or other hardware at or near the fracture site prior to the fracture event. A finding of indeterminate was not permitted.

\section{Statistical methods}

The primary objective of the Extension is to evaluate the safety and tolerability of denosumab. Key secondary objectives include evaluation of the effects on BTMs and BMD, and the incidence of new vertebral and nonvertebral fractures. The protocol specifies that all endpoints will be analyzed according to the original randomized treatment group assignments in FREEDOM.

Safety analyses included participants who received $\geq 1$ dose of investigational product in the Extension and were based on the original randomized treatment assignments in FREEDOM. Therefore, in the current safety analyses, seven of the original placebo-group subjects who received one incorrect dose of denosumab in FREEDOM and were previously summarized in the denosumab group [1] were included in the FREEDOM placebo group. For consistency with previous reports of the FREEDOM Extension data [2, 3], the Medical Dictionary for Regulatory Activities v13.0 was used to code and report AEs for this publication. Descriptive analyses of AEs and serious adverse events (SAEs) included exposureadjusted subject incidence rates. Within each yearly or cumulative interval, the exposure-adjusted subject incidence was calculated as the number of subjects who experienced a particular AE divided by the total exposure time within the interval. A subject who experienced repeated episodes of the same AE within the yearly or cumulative interval of interest was counted only once for that interval. For example, a subject who experienced a common cold during years 1 and 3 was counted once in year 1 and once in year 3 for those yearly intervals, but only once for the 5-year cumulative interval.

Analyses of BTMs included participants who received $\geq 1$ dose of investigational product in the Extension and had observed values at the time points of interest. Results are presented as medians and interquartile ranges. Analyses of BMD percentage change from either FREEDOM or Extension baseline required observed values at the respective baseline and the time points of interest, and were conducted using a repeated measures model as described previously [2]. Least squares means with $95 \%$ confidence intervals were calculated and reported. In addition, BMD gains between consecutive DXA evaluations during the Extension were assessed.

Cumulative and yearly crude subject incidence of new vertebral fractures and Kaplan-Meier estimates of cumulative and yearly subject incidence of nonvertebral fractures were calculated.

Fracture and safety data from the FREEDOM parent study have been summarized to allow comparison with the Extension results. For BTMs and BMD, only data from women enrolled in the Extension are presented.

\section{Results}

Of the 7808 women enrolled in the FREEDOM study, 5928 (76\%) were eligible for enrollment in the Extension, and of these, 4550 (77\%) enrolled (2343 long-term, 2207 crossover) (Fig. 1). Of the women who entered the Extension, $66 \%$ in each group completed year 5 totaling 3004 participants. The percentage of women who discontinued the study and the reasons for discontinuation were similar between the two groups (Fig. 1). The demographics of the two groups were balanced at the start of the Extension [2]; Online Resource Table S1 summarizes characteristics for the long-term and cross-over groups at both the FREEDOM and Extension baselines. The mean (standard deviation) age for the women who completed year 5 of the Extension was 79.0 (4.7)years. Since these two groups have different durations of exposure to denosumab, the results from each group are described separately below. 
Fig. 1 Disposition of all participants through year 5 of the FREEDOM Extension. All women who completed FREEDOM (i.e., completed their 3 -year visit, did not discontinue investigation product, and did not miss more than one dose) were eligible to participate in the Extension. ${ }^{\mathrm{a}}$ Two women who discontinued denosumab also entered the Extension in the long-term denosumab group [2]

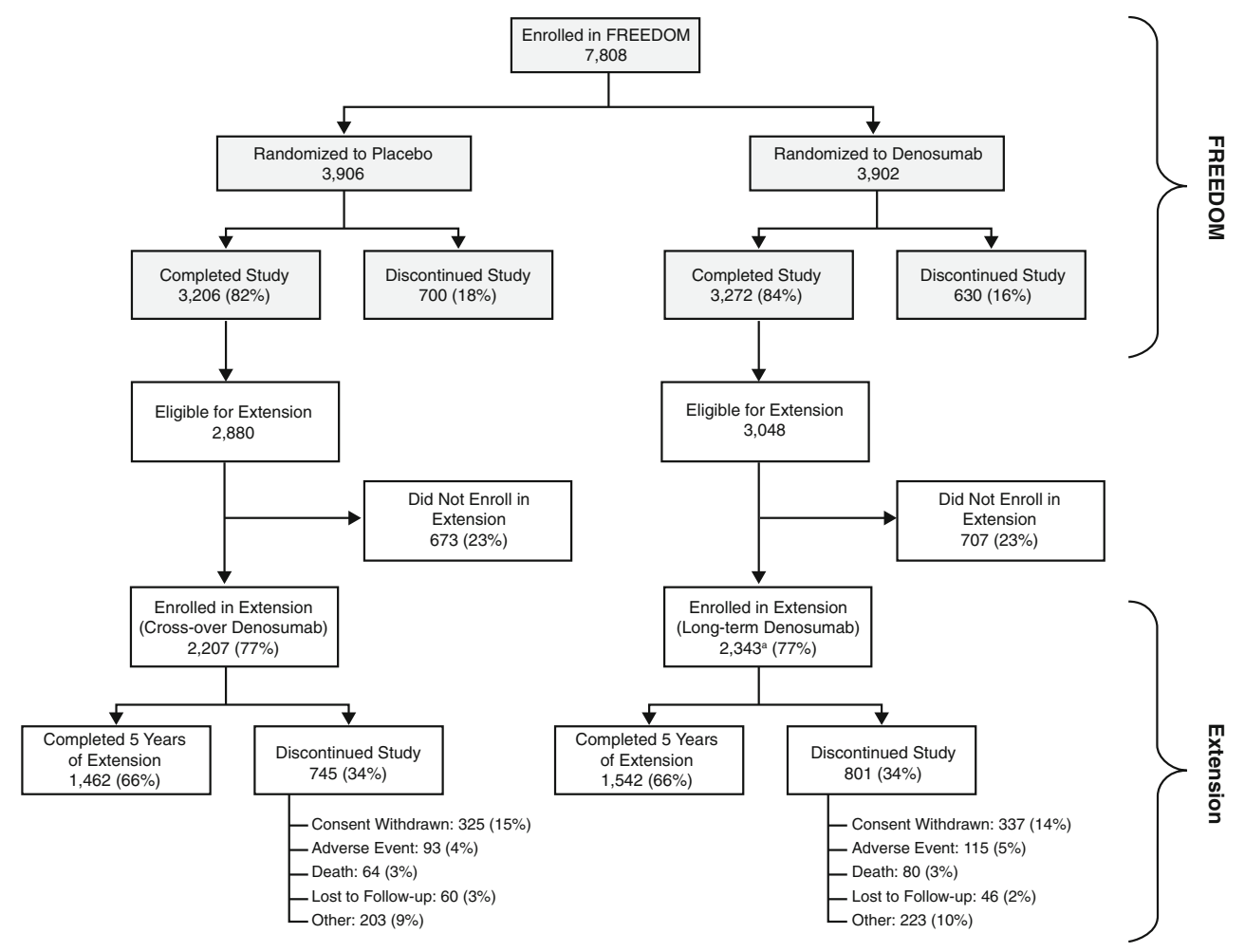

\section{Long-term group}

\section{$B T M s$ and $B M D$}

Following administration of denosumab at the beginning of the Extension, prompt decreases in serum CTx and P1NP concentrations were observed at month 1 , followed by a characteristic attenuation in the degree of reduction of remodeling through month 6, consistent with the FREEDOM parent study (Fig. 2). Through year 5 of the Extension, representing up to 8 years of therapy, denosumab treatment resulted in sustained reduction of serum CTx and P1NP levels.

During the Extension, mean percentage changes from FREEDOM baseline in lumbar spine, total hip, and femoral neck BMD at each time point were significantly greater than those observed at the previous time point (Online Resource Table S2). Mean percentage changes in BMD from FREEDOM through Extension year 5, totaling up to 8 years of treatment, were increases of $18.4 \%$ at the lumbar spine, $8.3 \%$ at the total hip, $7.8 \%$ at the femoral neck, and $3.5 \%$ at the $1 / 3$ radius (all $p<0.05$ ) (Fig. 3).

\section{Fractures}

Among subjects who entered the Extension and had at least one subsequent X-ray assessment for new vertebral fracture, 116 women $(5.5 \%)$ in the long-term group had at least one new vertebral fracture by Extension year 5. Throughout the Extension, the annualized subject incidence of new vertebral fractures remained low $(1.5,1.3$, and $1.3 \%$ during years $4 / 5$, 6 , and 7/8 of denosumab treatment, respectively) (Fig. 4a and Online Resource Fig. S2). By Extension year 5, 133 women $(6.6 \%)$ had at least one nonvertebral fracture, and the yearly subject incidence remained low $(1.5,1.2,1.8,1.6$, and $0.7 \%$ during years $4,5,6,7$, and 8 of denosumab treatment, respectively) (Fig. 4b and Online Resource Fig. S2). Through year 5 of the Extension, the most common nonvertebral fracture sites in the long-term group were wrist $(n=57)$, rib $(n=17)$, hip (femoral neck or intertrochanteric) $(n=13)$, and ankle $(n=$ 12) ( $n$ is the number of affected women). The cumulative subject incidence of hip fractures during years 1 through 5 of the Extension was $0.7 \%$; the annualized incidence of hip fractures during Extension year 5 (totaling up to 8 years of denosumab treatment), when the mean age of the group was 79.0 years, was $0.2 \%$.

\section{AEs and SAEs}

The yearly exposure-adjusted subject incidence for all AEs during the Extension in the long-term group was similar to or lower than in the active treatment group during FREEDOM (Table 1). The yearly rates for malignancy and serious infections as well as for other AEs of interest per 100 subject-years showed no trend toward increase over time (Table 1). For AEs and SAEs where events were $<0.1$ per 100 subjects-years, yearly rates expressed per 10,000 subject-years are shown in Table 2. The cumulative exposure-adjusted subject incidence rates for all AEs, SAEs, and fatal AEs in the long-term group 


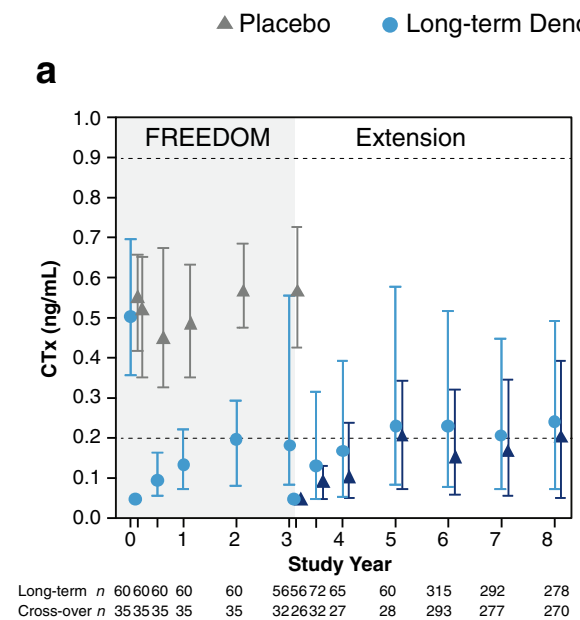

Fig. 2 Serum bone turnover markers during FREEDOM and the FREEDOM Extension. Concentrations of predose CTx (a) and P1NP (b) are shown. Dashed lines represent the premenopausal reference ranges: $0.20-0.90 \mathrm{ng} / \mathrm{mL}$ for $\mathrm{CTx}$ and $17.4-61.6 \mu \mathrm{g} / \mathrm{L}$ for P1NP. Data

b

during the Extension were 100.8, 10.7, and 0.8 per 100 subject-years, respectively (Online Resource Tables S3 and S4).

During 8 years of denosumab treatment, four subtrochanteric/diaphyseal femoral fractures occurred in the long-term group; of these, one was adjudicated as consistent with atypical diaphyseal femoral fracture. It

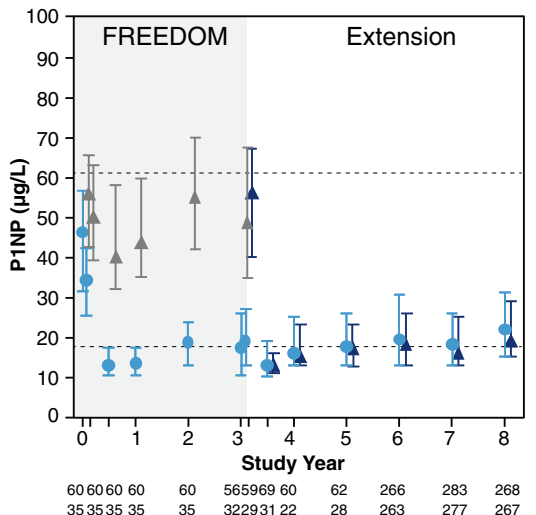

are median (interquartile range). $n=$ number of women with observed data. Time points shown include baseline, month 1 , and years $0.5,1,2$, 3,3 (day 10), 3.5, 4, 5, 6, 7, and 8. CTx serum C-terminal telopeptide of type 1 collagen, $P 1 N P$ serum procollagen type $1 \mathrm{~N}$-terminal propeptide

occurred in Extension year 4 after the participant received her 14th dose of denosumab. Initial treatment included internal fixation with an intramedullary nail. The participant developed hardware failure in the form of broken distal locking screws, which was detected 6 weeks after intramedullary rod placement. Union was
Fig. 3 Percentage change from FREEDOM baseline in BMD during FREEDOM and the FREEDOM Extension. Percentage change in BMD at the lumbar spine (a), total hip (b), femoral neck (c), and $1 / 3$ radius (d) is shown. Data are least squares means $(95 \% \mathrm{CI})$. ${ }^{*} p<0.05$ compared with the FREEDOM baseline; ${ }^{\dagger} p<0.05$ compared with the Extension baseline. $B M D$ bone mineral density, $C I$ confidence interval

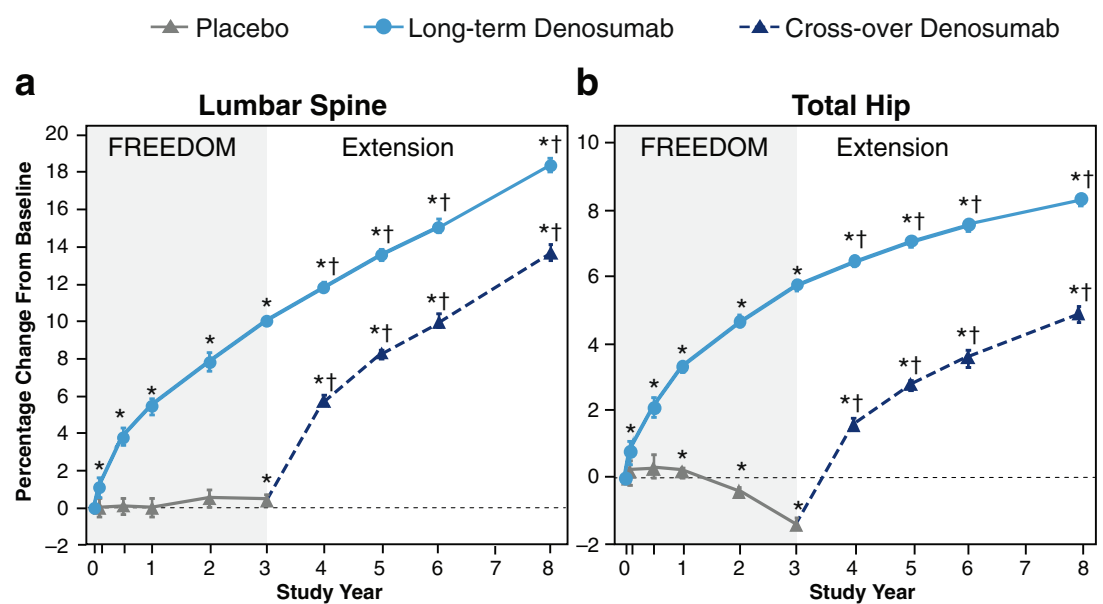

C

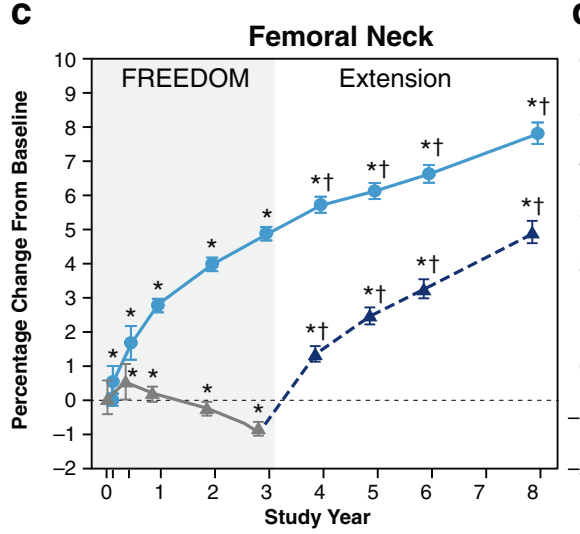

1/3 Radius

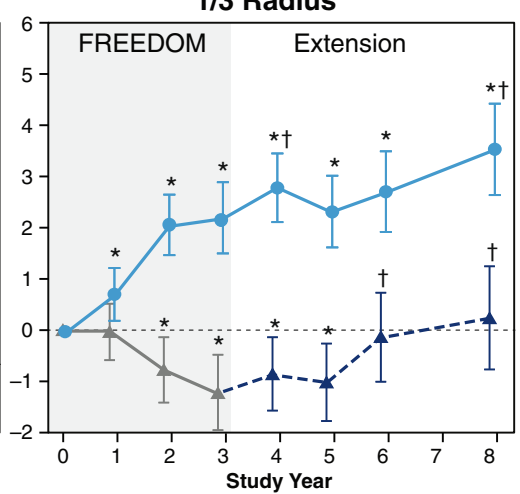


Fig. 4 Incidence of nonvertebral and new vertebral fractures during FREEDOM and the FREEDOM Extension. The yearly incidence of new vertebral and nonvertebral fractures in the long-term $(\mathbf{a}, \mathbf{b})$ and cross-over $(\mathbf{c}, \mathbf{d})$ groups are shown. For new vertebral fractures, percentages are crude incidence; lateral radiographs (lumbar and thoracic) were not obtained at Extension years 1 and 4 (long-term denosumab treatment years 4 and 7); $n=$ number of women with $\geq 1$ fracture; $N=$ number of women with a spine $\mathrm{X}$-ray evaluation during the time period of interest. ${ }^{\mathrm{a}}$ Annualized incidence (2-year incidence/2). For nonvertebral fractures, percentages are KaplanMeier estimates; $n=$ number of women with $\geq 1$ fracture; $N=$ number of women who were still on study at the beginning of each period

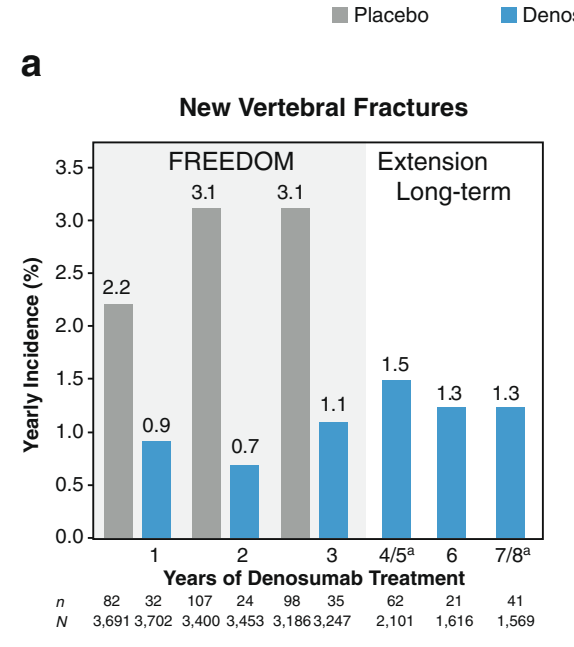

b

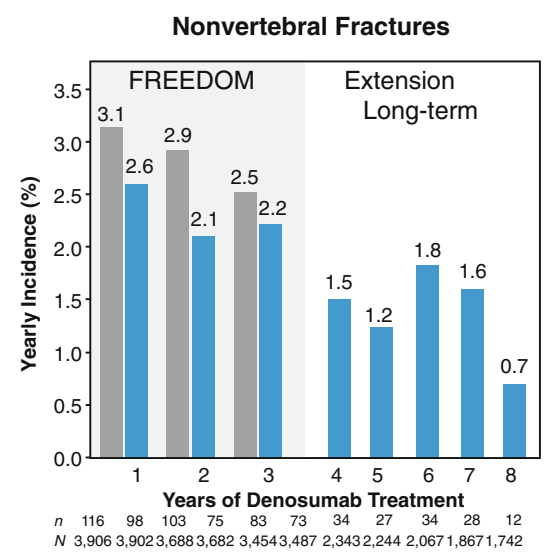

C

d

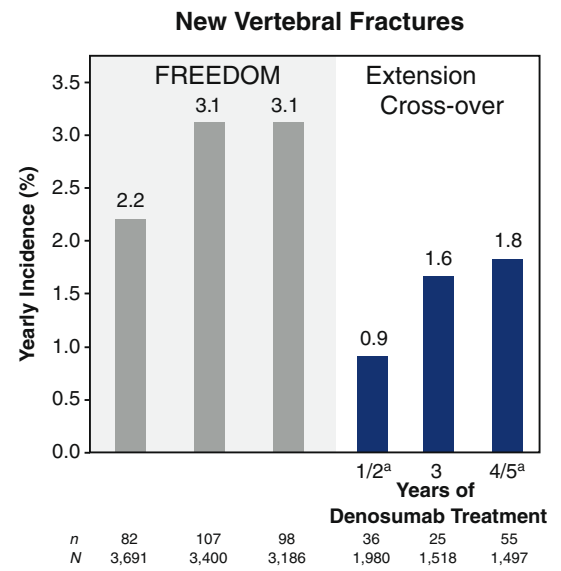

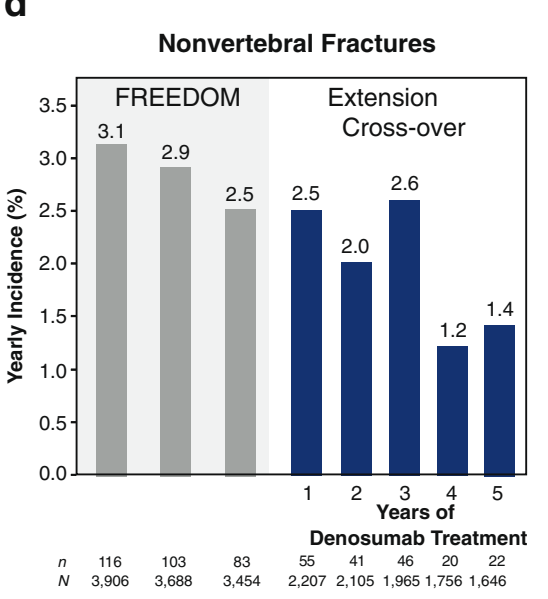

delayed but progressed after surgical revision was performed, approximately 20 months after detection of the broken screws. The participant has continued denosumab treatment and remained in the study. There were no cases of AFF in the long-term group during year 5 .

Through year 5 of the Extension, five oral events have been adjudicated as consistent with ONJ in the long-term group. Of the four previously reported cases [3], one event previously reported as resolved is ongoing at the time of this report. The fifth event occurred in the fourth year of the Extension, after the participant received her 13th dose of denosumab; denosumab was discontinued and the event has resolved. No additional ONJ events were reported during year 5 .

\section{Cross-over group}

\section{BTMs and BMD}

In the cross-over group, the median concentrations of serum CTx and P1NP were rapidly reduced after the initial administration of denosumab (day 10 and month 6 of the Extension, respectively) and were similar to those observed in the denosumab group in the FREEDOM parent study (Fig. 2). The reductions in both markers were also sustained through 5 years of denosumab treatment and, over time, the bone turnover profiles were consistent with the long-term group during their first 5 years of denosumab exposure.

BMD increased rapidly in the cross-over group during the first year of denosumab treatment, and measurements at the lumbar spine, total hip, and femoral neck continued to increase significantly at each time point measured compared to the previous time point through 5 years of treatment (Online Resource Table S2). The mean percentage changes in BMD from Extension baseline were $13.1 \%$ at the lumbar spine, $6.2 \%$ at the total hip, $5.7 \%$ at the femoral neck, and $1.5 \%$ at the $1 / 3$ radius (all $p<0.05$ ) (Fig. 3). These values largely replicated those observed in the long-term group after 5 years of denosumab exposure, which were $13.7 \%$ at the lumbar spine, $7.0 \%$ at the total hip, $6.1 \%$ at the femoral neck, and $2.3 \%$ at the $1 / 3$ radius (all $p<0.05$ ) [2]. 
Table 1 Yearly exposure-adjusted subject incidence of adverse events per 100 subject-years for long-term denosumab participants

\begin{tabular}{|c|c|c|c|c|c|c|c|c|c|}
\hline \multirow[b]{2}{*}{ Denosumab exposure } & \multicolumn{4}{|c|}{$\begin{array}{l}\text { FREEDOM (years 1-3) } \\
\text { Denosumab }\end{array}$} & \multicolumn{5}{|c|}{$\begin{array}{l}\text { FREEDOM Extension (years 1-5) } \\
\text { Long-term denosumab }\end{array}$} \\
\hline & $N=$ & $\begin{array}{l}\text { Year } 1 \\
3879\end{array}$ & $\begin{array}{l}\text { Year } 2 \\
3682\end{array}$ & $\begin{array}{l}\text { Year } 3 \\
3487\end{array}$ & $\begin{array}{l}\text { Year } 4 \\
2343\end{array}$ & $\begin{array}{l}\text { Year } 5 \\
2244\end{array}$ & $\begin{array}{l}\text { Year } 6 \\
2067\end{array}$ & $\begin{array}{l}\text { Year } 7 \\
1867\end{array}$ & $\begin{array}{l}\text { Year } 8 \\
1742\end{array}$ \\
\hline All adverse events & & 188.5 & 154.9 & 131.6 & 134.8 & 116.9 & 114.2 & 120.8 & 106.3 \\
\hline Infections & & 37.7 & 31.9 & 31.0 & 30.3 & 26.8 & 27.5 & 28.9 & 26.9 \\
\hline Malignancies & & 1.8 & 1.5 & 2.2 & 2.0 & 2.4 & 2.0 & 2.7 & 1.7 \\
\hline Eczema & & 1.6 & 1.1 & 0.9 & 1.0 & 1.3 & 1.0 & 0.7 & 0.7 \\
\hline Hypocalcemia & & 0 & 0 & 0 & 0 & $<0.1$ & 0 & $<0.1$ & 0 \\
\hline Pancreatitis & & $<0.1$ & $<0.1$ & $<0.1$ & 0 & $<0.1$ & 0.2 & $<0.1$ & 0.1 \\
\hline Serious adverse events & & 11.8 & 11.2 & 12.0 & 10.8 & 13.0 & 12.3 & 15.4 & 11.3 \\
\hline Infections & & 1.5 & 1.6 & 1.6 & 1.3 & 1.2 & 1.7 & 2.4 & 1.2 \\
\hline Cellulitis or erysipelas & & 0.1 & $<0.1$ & 0.2 & $<0.1$ & $<0.1$ & 0.1 & 0 & 0.2 \\
\hline Fatal adverse events & & 0.6 & 0.6 & 0.7 & 0.4 & 0.8 & 0.9 & 1.3 & 0.7 \\
\hline Osteonecrosis of the jaw & & 0 & 0 & 0 & 0 & 0 & 0.2 & $<0.1$ & 0 \\
\hline Atypical femoral fracture & & 0 & 0 & 0 & 0 & 0 & 0 & $<0.1$ & 0 \\
\hline
\end{tabular}

Analyses were based on the original randomized treatments in FREEDOM. All adverse and serious adverse events were coded using MedDRA v13.0 $N$ number of women who received $\geq 1$ dose of investigational product in FREEDOM or the Extension and were on study at the beginning of the yearly interval

\section{Fractures}

During the fifth year of the Extension, totaling up to 5 years of denosumab treatment, fracture rates remained low in the cross-over group; 110 women in the cross-over group $(5.5 \%)$ had at least one new vertebral fracture. These low rates are similar to those observed during the first 5 years of denosumab treatment in the long-term group $(4.0 \%)$. In addition, the annualized subject incidence of new vertebral fracture remained low after the initiation of denosumab $(0.9,1.6$, and $1.8 \%$ during years $1 / 2,3$, and $4 / 5$ of denosumab treatment, respectively)
(Fig. 4c and Online Resource Fig. S2). One hundred and seventy-five women $(9.2 \%)$ had at least one nonvertebral fracture through year 5 of the Extension, consistent with the cumulative subject incidence observed over the first 5 years of denosumab treatment in the long-term group (7.9\%). Likewise, the yearly subject incidence remained low $(2.5,2.0,2.6,1.2$, and $1.4 \%$ during years $1,2,3,4$, and 5 of denosumab treatment, respectively) (Fig. 4d and Online Resource Fig. S2). Through year 5 of the Extension, the most common nonvertebral fracture sites in the cross-over group were wrist $(n=76)$, hip (femoral neck or intertrochanteric) $(n=22)$, ankle $(n=19)$, humerus

Table 2 Yearly exposure-adjusted subject incidence of adverse events per 10,000 subject-years for long-term denosumab participants where events of interest were $<0.1$ per 100 subject-years

\begin{tabular}{|c|c|c|c|c|c|c|c|c|c|}
\hline \multirow[b]{2}{*}{ Denosumab exposure } & \multicolumn{4}{|c|}{$\begin{array}{l}\text { FREEDOM (years 1-3) } \\
\text { Denosumab }\end{array}$} & \multicolumn{5}{|c|}{$\begin{array}{l}\text { FREEDOM Extension (years 1-5) } \\
\text { Long-term denosumab }\end{array}$} \\
\hline & $N=$ & $\begin{array}{l}\text { Year } 1 \\
3879\end{array}$ & $\begin{array}{l}\text { Year } 2 \\
3682\end{array}$ & $\begin{array}{l}\text { Year } 3 \\
3487\end{array}$ & $\begin{array}{l}\text { Year } 4 \\
2343\end{array}$ & $\begin{array}{l}\text { Year } 5 \\
2244\end{array}$ & $\begin{array}{l}\text { Year } 6 \\
2067\end{array}$ & $\begin{array}{l}\text { Year } 7 \\
1867\end{array}$ & $\begin{array}{l}\text { Year } 8 \\
1742\end{array}$ \\
\hline Hypocalcemia & & 0 & 0 & 0 & 0 & 9.2 & 0 & 5.6 & 0 \\
\hline Pancreatitis & & 5.3 & 5.6 & 8.7 & 0 & 4.6 & 15.6 & 5.6 & 12.2 \\
\hline Serious cellulitis or erysipelas & & 10.6 & 2.8 & 23.3 & 8.7 & 4.6 & 10.4 & 0 & 18.3 \\
\hline Osteonecrosis of the jaw & & 0 & 0 & 0 & 0 & 0 & 20.8 & 5.6 & 0 \\
\hline Atypical femoral fracture & & 0 & 0 & 0 & 0 & 0 & 0 & 5.6 & 0 \\
\hline
\end{tabular}

Analyses were based on the original randomized treatments in FREEDOM. All adverse and serious adverse events were coded using MedDRA v13.0 $N$ number of women who received $\geq 1$ dose of investigational product in FREEDOM or the Extension and were on study at the beginning of the yearly interval 
$(n=15)$, and rib $(n=14)$ ( $n$ is the number of affected women). The cumulative subject incidence of hip fractures during years 1 through 5 of the Extension was $1.1 \%$.

\section{AEs and SAEs}

Overall, the AE rates in the cross-over group confirmed those previously reported for the corresponding time period in the long-term group [2]. The yearly exposure-adjusted subject incidence for all AEs in the cross-over group during the Extension was similar to or lower than that of the placebo group during FREEDOM (Table 3). The yearly rates per 100 subject-years showed no increase over time. For AEs and SAEs where events were $<0.1$ per 100 subjects-years, yearly rates expressed per 10,000 subject-years are shown in Table 4 . The cumulative exposure-adjusted subject incidence rates of all AEs, SAEs, and fatal AEs in the cross-over group during the Extension were 99.7, 10.2, and 0.7 per 100 subject-years, respectively (Online Resource Tables S3 and S4).

During 5 years of denosumab treatment, three diaphyseal femoral fractures occurred in the cross-over group. Of these, one fracture was adjudicated as consistent with AFF, as previously reported [3]. There were no reports of AFF during year 5 of the Extension in the cross-over group.

Through year 5 of the Extension, three oral events have been adjudicated as consistent with ONJ in the cross-over group. Two of these events resolved as previously reported [3]. The third event occurred in year 4 of the Extension, after the participant received her eighth dose of denosumab. The participant discontinued the study. Hence, the outcome of the case is unknown. There were no ONJ cases during Extension year 5 .

\section{Discussion}

The FREEDOM Extension is a large ongoing study to evaluate the long-term benefit/risk of denosumab treatment for osteoporosis. These data address important considerations in interpreting the safety and efficacy of a new therapeutic class of treatment for long-term use in a chronic condition in an aging population. The study design incorporates a long-term treatment group to characterize the effects of up to 10 years of exposure, and a cross-over treatment cohort to further characterize and confirm assessments of efficacy and safety findings from extended denosumab administration, with a 3-year delay.

The primary purpose of this study is to evaluate the longterm safety of denosumab. The year-by-year results for FREEDOM and its Extension, presented here for the first time, demonstrate that the safety profile of denosumab has remained consistent over 8 years of treatment. Notably, in this aging population, the subject incidence of AEs such as serious infection, cellulitis, and eczema remained low with no evidence of increased frequency through 8 years of denosumab exposure, as did infrequent events. Bone safety events of ONJ and AFF were observed, and the rates remained low. Through

Table 3 Yearly exposure-adjusted subject incidence of adverse events per 100 subject-years for cross-over denosumab participants

\begin{tabular}{|c|c|c|c|c|c|c|c|c|c|}
\hline \multirow[b]{2}{*}{ Denosumab exposure } & \multicolumn{4}{|c|}{$\begin{array}{l}\text { FREEDOM (years 1-3) } \\
\text { Placebo }\end{array}$} & \multicolumn{5}{|c|}{$\begin{array}{l}\text { FREEDOM Extension (years 1-5) } \\
\text { Cross-over denosumab }^{\mathrm{a}}\end{array}$} \\
\hline & $N=$ & $-\overline{3883}$ & - & $\begin{array}{l}- \\
3454\end{array}$ & $\begin{array}{l}\text { Year } 1 \\
2206\end{array}$ & $\begin{array}{l}\text { Year } 2 \\
2105\end{array}$ & $\begin{array}{l}\text { Year } 3 \\
1965\end{array}$ & $\begin{array}{l}\text { Year } 4 \\
1756\end{array}$ & $\begin{array}{l}\text { Year } 5 \\
1646\end{array}$ \\
\hline All adverse events & & 189.5 & 156.3 & 132.8 & 132.1 & 112.3 & 109.8 & 122.5 & 100.1 \\
\hline Infections & & 38.6 & 33.9 & 31.7 & 30.8 & 27.5 & 26.0 & 27.6 & 24.7 \\
\hline Malignancies & & 1.8 & 1.6 & 1.5 & 1.9 & 1.6 & 2.1 & 2.6 & 2.1 \\
\hline Eczema & & 0.8 & 0.5 & 0.6 & 1.0 & 0.9 & 1.1 & 1.2 & 1.0 \\
\hline Hypocalcemia & & $<0.1$ & 0 & $<0.1$ & 0.2 & $<0.1$ & 0 & $<0.1$ & 0.2 \\
\hline Pancreatitis & & $<0.1$ & $<0.1$ & 0 & $<0.1$ & 0 & $<0.1$ & 0 & 0 \\
\hline Serious adverse events & & 11.7 & 11.9 & 10.8 & 12.4 & 12.2 & 12.3 & 12.3 & 12.3 \\
\hline Infections & & 1.1 & 1.4 & 1.4 & 1.6 & 1.5 & 1.1 & 1.6 & 1.3 \\
\hline Cellulitis or erysipelas & & 0 & 0 & $<0.1$ & 0 & $<0.1$ & 0 & $<0.1$ & 0.1 \\
\hline Fatal adverse events & & 0.8 & 0.8 & 1.0 & 0.9 & 0.6 & 0.5 & 0.7 & 0.8 \\
\hline Osteonecrosis of the jaw & & 0 & 0 & 0 & 0 & 0.1 & 0 & $<0.1$ & 0 \\
\hline Atypical femoral fracture & & 0 & 0 & 0 & 0 & 0 & $<0.1$ & 0 & 0 \\
\hline
\end{tabular}

Analyses were based on the original randomized treatments in FREEDOM. All adverse and serious adverse events were coded using MedDRA v13.0 $N$ number of women who received $\geq 1$ dose of investigational product in FREEDOM or the Extension and were on study at the beginning of the yearly interval

${ }^{a}$ One cross-over subject did not receive investigational product in the Extension and therefore was excluded from the safety analysis 
Table 4 Yearly exposure-adjusted subject incidence of adverse events per 10,000 subject-years for cross-over denosumab participants where events of interest were $<0.1$ per 100 subject-years

\begin{tabular}{|c|c|c|c|c|c|c|c|c|c|}
\hline \multirow[b]{2}{*}{ Denosumab exposure } & \multicolumn{4}{|c|}{$\begin{array}{l}\text { FREEDOM (years 1-3) } \\
\text { Placebo }\end{array}$} & \multicolumn{5}{|c|}{$\begin{array}{l}\text { FREEDOM Extension (years 1-5) } \\
\text { Cross-over denosumab }^{\mathrm{a}}\end{array}$} \\
\hline & $N=$ & $\begin{array}{l}- \\
3883\end{array}$ & $\begin{array}{l}- \\
3687\end{array}$ & $\begin{array}{l}- \\
3454\end{array}$ & $\begin{array}{l}\text { Year } 1 \\
2206\end{array}$ & $\begin{array}{l}\text { Year } 2 \\
2105\end{array}$ & $\begin{array}{l}\text { Year } 3 \\
1965\end{array}$ & $\begin{array}{l}\text { Year } 4 \\
1756\end{array}$ & $\begin{array}{l}\text { Year } 5 \\
1646\end{array}$ \\
\hline Hypocalcemia & & 5.3 & 0 & 3.0 & 23.2 & 4.9 & 0 & 5.9 & 19.1 \\
\hline Pancreatitis & & 5.3 & 2.8 & 0 & 4.6 & 0 & 5.5 & 0 & 0 \\
\hline Serious cellulitis or erysipelas & & 0 & 0 & 3.0 & 0 & 4.9 & 0 & 5.9 & 12.7 \\
\hline Osteonecrosis of the jaw & & 0 & 0 & 0 & 0 & 9.8 & 0 & 5.9 & 0 \\
\hline Atypical femoral fracture & & 0 & 0 & 0 & 0 & 0 & 5.5 & 0 & 0 \\
\hline
\end{tabular}

Analyses were based on the original randomized treatments in FREEDOM. All adverse and serious adverse events were coded using MedDRA v13.0 $N$ number of women who received $\geq 1$ dose of investigational product in FREEDOM or the Extension and were on study at the beginning of the yearly interval

${ }^{a}$ One cross-over subject did not receive investigational product in the Extension and therefore was excluded from the safety analysis

Extension year 5, eight events of ONJ and two events of AFF were confirmed by adjudicators. The cumulative exposureadjusted subject incidence rates during the Extension (years $1-5)$, including both the long-term and cross-over groups, were 4.2 per 10,000 subject-years for ONJ and 1.0 per 10 , 000 subject-years for AFF.

Denosumab's mechanism of action, which prevents the generation, proliferation, and survival of osteoclasts, results in a marked reduction of bone resorption during most of the dosing period, though the pharmacodynamic profile shows some release of inhibition toward the end of the dosing interval. The BTMs described in the study were measured at the end of the dosing intervals when the effect of denosumab has been shown to wane, and undetectable values were imputed at the lower limit of detection, which may overestimate levels. Thus, reported levels of serum CTx may not fully reflect the maximal antiresorptive effect that occurs during much of the dosing interval [6]. Bone formation markers also are reduced. This turnover pattern was associated with a finding that is different from other antiresorptive therapies to date, namely a continuous progressive and significant increase in BMD. In both groups, markers of bone resorption remained low and BMD at the lumbar spine, total hip, and femoral neck continued to increase at each time point measured over the course of the Extension. BMD also continued to increase at the 1/3 radius in both the long-term and cross-over groups. These gains are noteworthy, as the radius has been shown to be minimally responsive to most osteoporosis treatments [9-11].

The overall effect of 5 years of denosumab treatment on BMD was similar between the two groups. Furthermore, the effects on BMD in the long-term group were consistent with the 8 -year gains reported in the lumbar spine and proximal femur in the phase 2 denosumab dose-ranging extension study [12]. The clinical significance of the BMD increases is supported by the associated sustained effects on fracture rates in both the long- term and cross-over groups during the Extension. The exact mechanism(s) underlying this pattern of BMD increases throughout the skeleton remains to be fully elucidated [3].

Hypotheses based on previous observations may help to explain the progressive gains in BMD without apparent therapeutic plateau and the low fracture incidence over time. As is known to occur with other antiresorptives, the early increase in BMD is at least partly due to the closing of the remodeling space and the subsequent secondary mineralization of bone apposed during the formation phase of existing remodeling units. However, such effects would be self-limited, and resultant BMD and mass gains would be expected to approach a plateau after 2 to 4 years $[13,14]$. With denosumab, this plateau in BMD has not been observed. Denosumab has been shown to decrease cortical porosity [15]. Furthermore, transient increases in parathyroid hormone following dosing have been documented [16]. Most recently, modeling-based bone formation was observed in the femoral neck of ovariectomized cynomolgus monkeys treated with denosumab for 16 months at a dose that fully inhibited remodeling [17]. That observation, which remains to be reproduced in humans, raises the possibility that denosumab does not prevent modeling and that ongoing modeling-based bone formation in a setting of maximally reduced resorption could contribute to the continued increases in bone mass. This is of particular clinical relevance because gains in BMD and mass, resulting from modeling in areas of high biomechanical stresses, could improve bone strength and reduce fracture risk $[18,19]$.

A significant limitation of this study is the lack of an ongoing placebo group, and these analyses cannot substitute for the results of a randomized placebo-controlled study. The placebo phase was terminated with the end of the core trial for ethical reasons. The previously reported virtual twin modeling approach addressed this limitation [2,3], as does the comparison of baseline characteristics of the Extension participants 
with the original study population. A per-protocol, post-hoc analysis of the rates of nonvertebral fractures through Extension year 4, limited to individuals who entered the Extension, has also been performed and is the subject of a separate report [20].

Subject attrition is also an important consideration in interpretation of safety and efficacy results from long-term followup studies. A series of analyses were recently presented that compared FREEDOM and Extension populations at baseline and in the Extension to assess potential selection bias that might influence long-term treatment fracture outcomes [21]. Increasing age and incident fracture were not deterrents to continued study participation, suggesting that safety and efficacy outcomes reflected denosumab treatment effect.

In conclusion, data from this ongoing long-term Extension study showed that continued treatment of women with postmenopausal osteoporosis with denosumab for up to 8 years resulted in sustained reduction of bone turnover, continued gains in BMD at each time point measured, and continued low yearly fracture incidence. Furthermore, the women in the cross-over group responded to 5 years of denosumab treatment similarly to the women in the long-term group during their first 5 years of denosumab treatment. The overall safety profile of denosumab in this ongoing study remains consistent over time, indicating a favorable balance between benefit and risk through 8 years of treatment.

Acknowledgments Lisa Humphries, Ph.D., of Amgen Inc. provided medical writing assistance. Funding for this study was provided by Amgen Inc.

Conflicts of interest S. Papapoulos has served as a consultant for Axsome, Merck, and Novartis, and as a speaker for Amgen Inc., GlaxoSmithKline, and Merck. K. Lippuner has served as a board member for Amgen Inc., Lilly, MSD, and UCB. C. Roux has served as a board member for Amgen Inc. and MSD; as a consultant and speaker for Amgen Inc., Eli Lilly, and MSD; and has received grants from Bongrain, Eli Lilly, and MSD. C.J.F. Lin, N.S. Daizadeh, A. Wang, M. Gavin, C. Libanati, and R.B. Wagman are employees and shareholders of Amgen Inc. D.L. Kendler has served as a consultant for Amgen Inc., Eli Lilly, Merck, and Pfizer; as a speaker for Amgen Inc. and Eli Lilly; and has received grants from Amgen Inc., Astellas, AstraZeneca, and Eli Lilly. E.M. Lewiecki has served as a board member for the National Osteoporosis Foundation and the International Society for Clinical Densitometry; as a consultant for Amgen Inc., AgNovos Healthcare, Alexion, Eli Lilly, Merck, Radius Health, and Theranova; and has received grants from Amgen Inc., Eli Lilly, and Merck.

M.L. Brandi has served as a board member for Amgen Inc., Alexion, Eli Lilly, and Servier; as a consultant for NPS and Servier; as a speaker for Amgen Inc., Alexion, Bruno Farmaceutici, MSD, Servier, and SpA; and has received grants from Amgen Inc., Alexion, Eli Lilly, Bruno Farmaceutici, MSD, Servier, Shire, and SpA. E. Czerwiński has served as a speaker for Amgen Inc. and Servier. E. Franek has served as a speaker for Amgen Inc., MSD, Novartis, and Servier. P. Lakatos has served as a board member for Amgen Inc., Novartis, and Servier, and as a speaker for Amgen Inc., MSD, Novartis, Roche, and Servier. C. Mautalen and S. Jensen have no conflicts of interest to declare. S. Minisola has served on advisory boards for Eli Lilly and Merck Sharp \& Dohme, and as a speaker for Abiogen, Amgen Inc., Bruno Farmaceutici, Eli Lilly, Italfarmaco, and Merck Sharp \& Dohme.

J-Y. Reginster has served on advisory boards and/or as a consultant for Amgen Inc., GlaxoSmithKline, Lilly, Merckle, Negma, Novartis, NPS, Nycomed, Roche, Servier, Theramex, UCB, and Wyeth; as a speaker for Analis, Ebewee Pharma, Genevrier, GlaxoSmithKline, IBSA, Lilly, Merck Sharp \& Dohme, Nolver, Novartis, NovoNordisk, Nycomed, Roche, Rottapharm, Servier, Teijin, Teva, Theramex, and Zodiac; and has received grants from Amgen Inc., Bristol Myers Squibb, GlaxoSmithKline, Lilly, Merck Sharp \& Dohme, Novartis, Roche, Rottapharm, Servier, and Teva. H. G. Bone has served as a consultant for Amgen Inc., Merck, Novartis, and Tarsa; as a speaker for Amgen Inc., and Merck; and has received grants from Amgen Inc., Merck, Novartis, and Tarsa.

Ethical approval This study was approved by an institutional review board and/or the ethics committee for each institutional site and was conducted in accordance with the Declaration of Helsinki. All study participants provided their written informed consent.

Open Access This article is distributed under the terms of the Creative Commons Attribution-NonCommercial 4.0 International License (http://creativecommons.org/licenses/by-nc/4.0/), which permits any noncommercial use, distribution, and reproduction in any medium, provided you give appropriate credit to the original author(s) and the source, provide a link to the Creative Commons license, and indicate if changes were made.

\section{References}

1. Cummings SR, San Martin J, McClung MR, Siris ES, Eastell R, Reid IR, Delmas P et al (2009) Denosumab for prevention of fractures in postmenopausal women with osteoporosis. N Engl J Med 361:756-765

2. Papapoulos S, Chapurlat R, Libanati C, Brandi ML, Brown JP, Czerwinski E, Krieg MA et al (2012) Five years of denosumab exposure in women with postmenopausal osteoporosis: results from the first two years of the freedom extension. J Bone Miner Res 27:694-701

3. Bone HG, Chapurlat R, Brandi ML, Brown JP, Czerwinski E, Krieg MA, Mellstrom D et al (2013) The effect of three or six years of denosumab exposure in women with postmenopausal osteoporosis: results from the freedom extension. J Clin Endocrinol Metab 98: 4483-4492

4. Keaveny TM, McClung MR, Genant HK, Zanchetta JR, Kendler D, Brown JP, Goemaere S et al (2014) Femoral and vertebral strength improvements in postmenopausal women with osteoporosis treated with denosumab. J Bone Miner Res 29:158-165

5. Austin M, Yang YC, Vittinghoff E, Adami S, Boonen S, Bauer DC, Bianchi G et al (2012) Relationship between bone mineral density changes with denosumab treatment and risk reduction for vertebral and nonvertebral fractures. J Bone Miner Res 27:687-693

6. Eastell R, Christiansen C, Grauer A, Kutilek S, Libanati C, McClung MR, Reid IR et al (2011) Effects of denosumab on bone turnover markers in postmenopausal osteoporosis. J Bone Miner Res 26:530-537

7. Genant HK, Wu CY, van Kuijk C, Nevitt MC (1993) Vertebral fracture assessment using a semiquantitative technique. J Bone Miner Res 8:1137-1148

8. Shane E, Burr D, Ebeling PR, Abrahamsen B, Adler RA, Brown TD, Cheung AM et al (2010) Atypical subtrochanteric and diaphyseal femoral fractures: report of a task force of the american society for bone and mineral research. J Bone Miner Res 25:2267-2294 
9. Finkelstein JS, Hayes A, Hunzelman JL, Wyland JJ, Lee H, Neer RM (2003) The effects of parathyroid hormone, alendronate, or both in men with osteoporosis. N Engl J Med 349:1216-1226

10. Bone HG, Hosking D, Devogelaer JP, Tucci JR, Emkey RD, Tonino RP, Rodriguez-Portales JA et al (2004) Ten years' experience with alendronate for osteoporosis in postmenopausal women. N Engl J Med 350:1189-1199

11. Devogelaer JP, Brown JP, Burckhardt P, Meunier PJ, Goemaere S, Lippuner K, Body JJ et al (2007) Zoledronic acid efficacy and safety over five years in postmenopausal osteoporosis. Osteoporos Int 18:1211-1218

12. McClung MR, Lewiecki EM, Geller ML, Bolognese MA, Peacock M, Weinstein RL, Ding B et al (2013) Effect of denosumab on bone mineral density and biochemical markers of bone turnover: 8-year results of a phase 2 clinical trial. Osteoporos Int 24:227-235

13. Black DM, Schwartz AV, Ensrud KE, Cauley JA, Levis S, Quandt SA, Satterfield S et al (2006) Effects of continuing or stopping alendronate after 5 years of treatment: the Fracture Intervention Trial Long-term Extension (FLEX): a randomized trial. JAMA 296:2927-2938

14. Black DM, Reid IR, Boonen S, Bucci-Rechtweg C, Cauley JA, Cosman F, Cummings SR et al (2012) The effect of 3 versus 6 years of zoledronic acid treatment of osteoporosis: a randomized extension to the HORIZON-Pivotal Fracture Trial (PFT). J Bone Miner Res 27:243-254

15. Seeman E, Delmas PD, Hanley DA, Sellmeyer D, Cheung AM, Shane E, Kearns A et al (2010) Microarchitectural deterioration of cortical and trabecular bone: differing effects of denosumab and alendronate. J Bone Miner Res 25:1886-1894
16. Seeman E, Libanati C, Austin M, Chapurlat R, Boyd SK, Zebaze R, Hanley DA et al (2011) The transitory increase in PTH following denosumab administration is associated with reduced intracortical porosity: a distinctive attribute of denosumab therapy. J Bone Miner Res 26(Suppl 1):S22

17. Ominsky M, Libanati C, Boyce RW, Kostenuik PJ, Baron R, Wagman RB, Dempster DW (2013) Continuous modeling-based bone formation: a novel mechanism that could explain the sustained increases in hip bone mineral density with denosumab treatment. J Bone Miner Res 28(Suppl 1):S504

18. Epker BN, Frost HM (1966) Periosteal appositional bone growth from age two to age seventy in man. A tetracycline evaluation. Anat Rec 154:573-577

19. Kontulainen S, Sievanen H, Kannus P, Pasanen M, Vuori I (2002) Effect of long-term impact-loading on mass, size, and estimated strength of humerus and radius of female racquetsports players: a peripheral quantitative computed tomography study between young and old starters and controls. J Bone Miner Res 17:2281-2289

20. Ferrari S, Adachi JD, Lippuner K, Zapalowski C, Miller PD, Reginster J-Y, Törring O et al (2015) Further reductions in nonvertebral fracture rate with long-term denosumab treatment in the freedom open-label extension and influence of hip bone mineral density after 3 years. Osteoporos Int (Accepted)

21. Adachi J, Ho P, Lin C, Bolognese M, Bone H, Hadji P, Papapoulos $S$ et al (2014) Evolution of subject characteristics in freedom and its extension for up to 8 years. J Bone Miner Res 29(Suppl 1):S270 